\title{
Segregated Handwritten Character Recognition using GLCM Features
}

\author{
V.C.Bharathi \\ Dept. of Computer Science and Engg., \\ Annamalai University
}

\author{
M. Kalaiselvi Geetha \\ Dept. of Computer Science and Engg., \\ Annamalai University
}

\begin{abstract}
Handwritten document recognition is an area of pattern recognition that has been showing impressive performance in the machine printed text. Handwritten document recognition is an intricate task to various writing styles of individual person. The system first identifies the contour in a handwritten document for segmentation and features are extracted from the segmented character. This paper uses GLCM(Gray Level Co-occurrence Matrix) for character recognition. Features of a character has been computed based on calculating the pairs of pixel with specific values and specified spatial relationship occurrence in an image. First order and second order textures are used to measure the intensity of the original pixels. Data were collected from different persons, and the system is trained using SVM with various writing styles. The proposed system achieves a maximum recognition accuracy of $95.2 \%$ with training and testing data using GLCM as features and SVM with RBF kernel function.
\end{abstract}

\section{Keywords:}

Handwritten Character Recognition, Segmentation, Gray Level Co-occurrence Matrix (GLCM), Support Vector Machine.

\section{INTRODUCTION}

Handwritten character recognition plays a vital role in the present world. It can solve difficult problems such as recognition of handwritten characters, written in different styles and makes humans profession easier. Machine simulation of handwritten recognition has become a topic of serious research. The main motivation for such an attempt was not only the challenge in handwritten character recognition but also the possibility of professional applications in which the data are presented on documents has to be transferred into editable text format. Human can recognize the character easily but making the computer system to recognize the handwritten character becomes difficult due to random variation of noise, varying styles, unvarying fonts and size. It involves several application areas like postal address reading, mail sorting, cheque reading, document conversion and libraries etc. Off-line character recognition is further divided into two parts, machine printed and handwritten character recognition. In handwritten character recognition, there are various problems as compared to machine printed document because of the different peoples have different styles of writing, the size of pen-tip and some people have skewness in their writing.

\subsection{Related Work}

Pankaj Kumawat et.al.1 propose character recognition method, which combines curvelet transform and invariant statistical features. HMM and SVM is used to classify the character. Gaurav Kumar et.al. 2 presents a discrete fourier transform method to measure the relevant shape information contained in a pattern to extract the features and classify the character using feed forward back propagation neural network. Rajib Lochan Das et.al. 3 propose offline English character recognition, to combine the global and local feature to classify the character using hidden Markov model. Mohammed Imrul Jubair et.al. 4 simplified method for handwritten character recognition from document image using morphological thinning operation. G Pirlo et.al. [5] 6] handwritten character recognition based on static and dynamic zoning topologies designed the regular grids that superimpose on the pattern images into regions of equal shape using voronoi based image zoning. Yusuf Perwej et.al. 7] propose machine recognition of handwritten character bounding boxes in individual character and unary operator that provide the sum of features for a character. Dayashankar et.al. 8 propose handwritten character recognition which combine conventional feature and gradient feature extraction to calculate horizontal and vertical gradients of pixels and classify the character with back propagation neural network.

\subsection{Outline of the work}

This paper deals with segregated handwritten character recognition using scanned document. The method has been evaluated with handwritten characters collected from different writers with various writing styles. GLCM has been computed for all the characters. First order and second order statistical texture features that are extracted from GLCM matrix as given as input to the SVM for recognition. The block diagram of the proposed work is shown in Fig 1 The rest of the paper is organised as follows, Section 2 descibes the preprocessing and segmentation. Feature extraction is described in section 3. Handwritten character recognition using SVM is presented in section 4. Section 5 shows the experimental results of our approach and section 6 concludes this work. 


\section{CHARACTER RECOGNITION}

Handwritten characters are collected from different writers, to ensure various writing styles. The written document is scanned and the scanned image is used for further analysis. The document image is initially binarized and morphological filtering is done on binarized image. Then the characters are segmented using intensity based threshold method. Segmented character is uniformly resized to $100 \times 100$ for future extraction. GLCM features are extracted from the segmented character image. Finally, LIBSVM is used to classify and recognize the character.

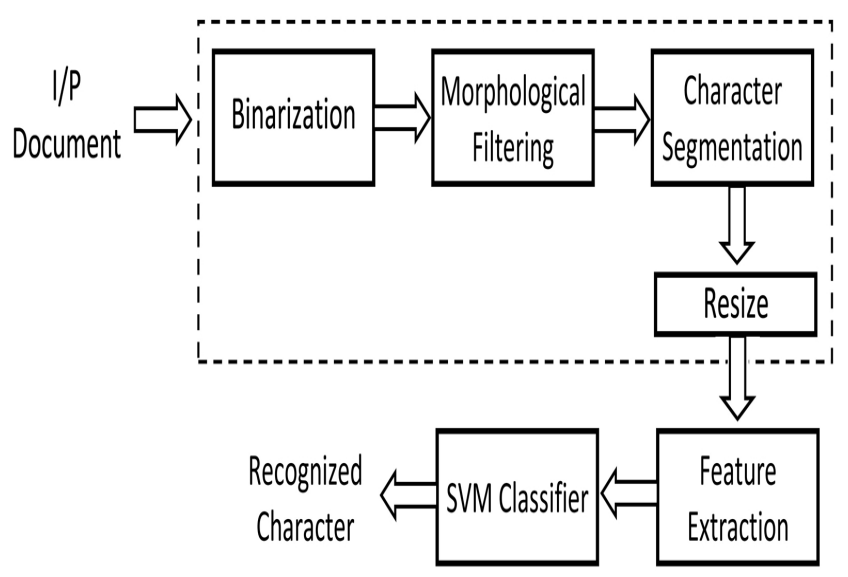

Fig. 1. System architecture.

\subsection{Pre-processing}

The pre-processing is a set of operation performed on the input document. The various tasks involved in the preprocessing stage are discussed here.

2.1.1 Binarization. Input document images collected from different writers may be in different colors. Hence the input document needs to be binarized for experimental use. Binarization is a process of converting an RGB image into a gray image and further into a binary image using intensity based thresholding method. In this work, threshold value 127 is used in binarization. The input image and the binarized image

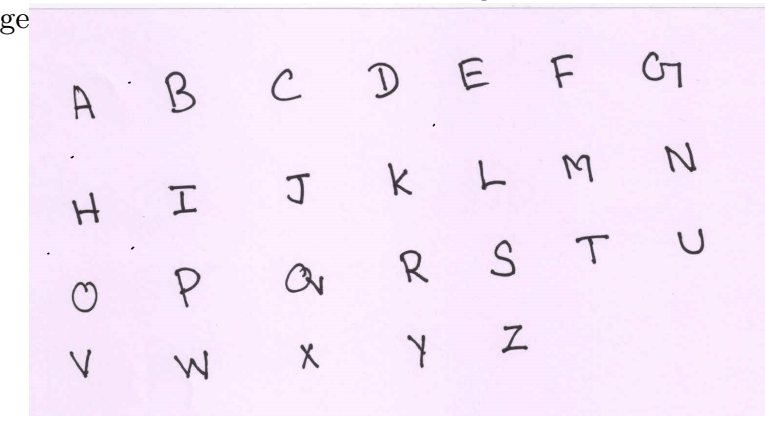

Fig. 2. Input Image.

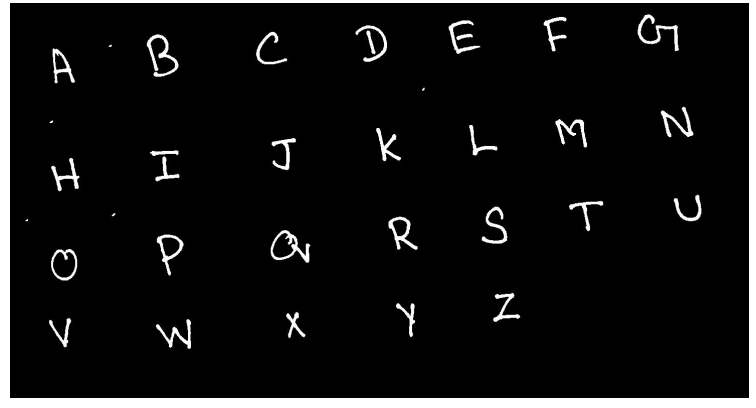

Fig. 3. Binarized image before morphological filtering.

2.1.2 Morphological Filtering. Binary image may contain numerous imperfections. Morphological filtering is applied over the binarized image to remove these imperfections. Binary area open method is a type of filtering which removes all the objects that contains fewer than 30 pixels of the input binary image and hence imperfections are removed as shown in Fig 4

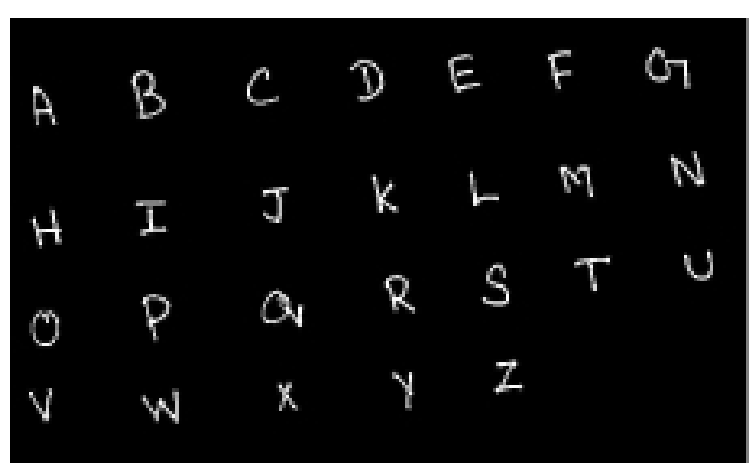

Fig. 4. Binarized image after morphological filtering.

2.1.3 Segmentation and Resizing. Segmentation is the process of extracting individual characters from the binarized document image. Segmentation is done by finding the connected components and placing a bounding box over it. The extracted individual characters which are of various sizes are uniformly resized. Fig 5 shows contours detected in the input document and the corresponding segmented characters are shown in Fig 6 and fed the character for feature extraction. 


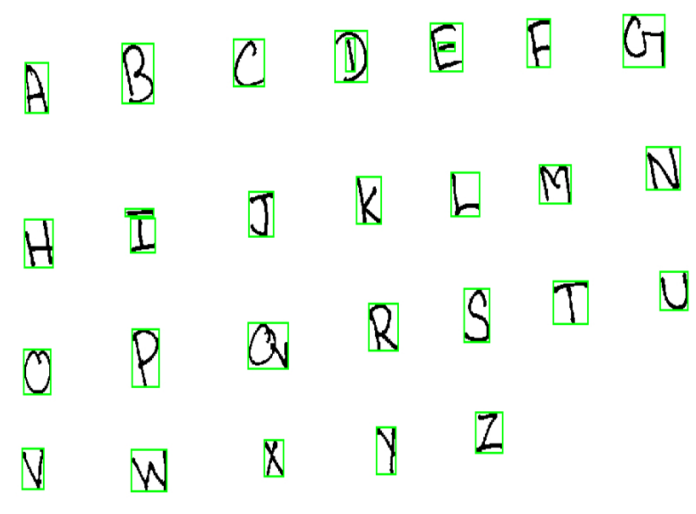

Fig. 5. Contours detected.

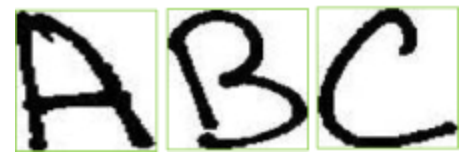

Fig. 6. Segmented character.

\section{FEATURE EXTRACTION}

The feature is a descriptive portion extracted from the input image. Recognizing the handwritten document character relies on the competent use of these features that provide discriminating information. The following subsection present the description of the features used in this study.

\subsection{First order Texture}

The first order texture feature such as mean and standard deviation are computed [9]. If $\mathrm{z}$ is a random variable indicate pixel intensity of $\mathrm{i}, \mathrm{p}(\mathrm{z})$ is the histogram of the intensity levels in a region, $\mathrm{G}$ is the number of possible gray level intensity, $\mu$ is the mean value.

The mean is a measure of average intensity:

$$
\operatorname{Mean}(\mu)=\sum_{i=0}^{G-1} z_{i} p\left(z_{i}\right)
$$

Standard deviation is a measure of average contrast.

$$
S D=\sqrt{\sum_{i=0}^{G-1}\left(z_{i}-\mu\right)^{2} p\left(z_{i}\right)}
$$

\subsection{Gray Level Co-Occurrence Matrix(GLCM)}

Texture analysis aims at finding a distinctive way of representing the essential characteristics of textures and represents them in simpler and unique form, so that they can be used for robust, accurate recognition. A geometric method of examining texture that considers the spatial relationship of pixels is the gray level co-occurrence matrix. The theory and techniques behind the Gray Level Co-occurrence Matrix (GLCM) method is presented in 10 . The GLCM functions characterize the texture of an image by calculating the pairs of pixel with specific values and specified spatial relationship occur in an image. In a graycomatrix, to calculate pixel with the intensity value $\mathrm{i}$ occurs in a specific spatial relationship to a pixel with the value $\mathrm{j}$. Each element $(\mathrm{i}, \mathrm{j})$ in GLCM specifies the number of times that the pixel with the value i occured adjacent to a pixel with value j. GLCM texture indicates the relationship between the reference and neighbor pixel of the gray level image at the various directions as shown in Fig 7 .

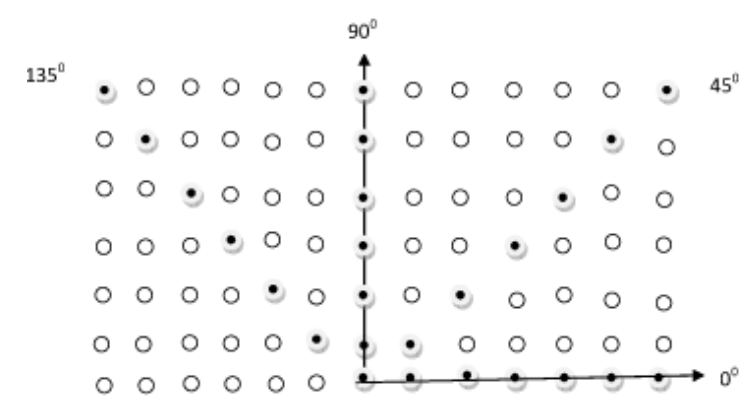

Fig. 7. GLCM matrix for 4 distances and 4 directions of adjaceny for texture features.
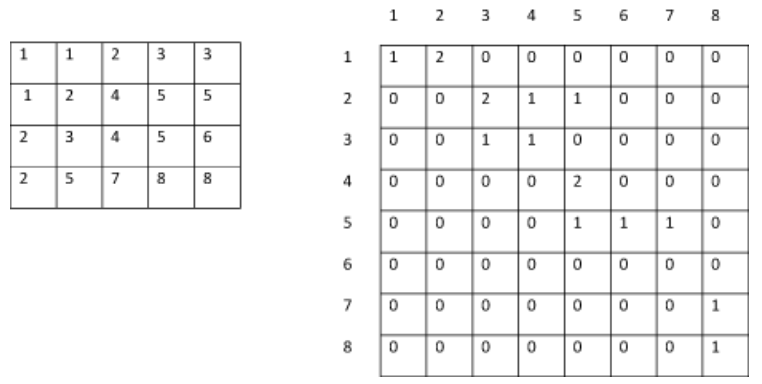

Fig. 8. GLCM Matrix for distance 1 and $0^{\circ}$ direction.

The adjacency can be specified to occur in each of four directions $0^{\circ}, 45^{\circ}, 90^{\circ}$ and $135^{\circ}$ degrees in a two dimensional square pixel image (directions horizontal, vertical, left and right diagonal). GLCM matrix stores the instance occurrences between adjacent pixels. Element $(1,2)$ in the GLCM contains the value 2 because there are two instances in the image are shown in Fig 8 Given an Image I, of size $\mathrm{NxN}$, the co-occurrence matrix $\mathrm{P}$ can be defined as

$p(i, j)=\sum_{x=1}^{G} \sum_{y=1}^{G} \begin{cases}1, & \text { if } I(x, y)=i \text { and } I\left(x+\Delta_{x}, y+\Delta_{y}\right)=j \\ 0, & \text { Otherwise }\end{cases}$ 
where the offset $\left(\Delta_{x}, \Delta_{y}\right)$, specifies the distance between the pixel of interest and its neighbour. i,j specifies intensity values of the image and $\mathrm{x}, \mathrm{y}$ are the spatial positions in the image I.

A number of texture features are extracted from the GLCM. In this paper, nine texture descriptors are used namely contrast, dissimilarity, entropy, sum of square variance, sum of average, sum variance, sum of entropy, difference variance and difference entropy. GLCM 9 dimensional sample features for various qualities and different styles as shown in Fig 9 .

Contrast(CON):

Measure of contrast or local intensity variation will favour contributions from $\mathrm{p}(\mathrm{i}, \mathrm{j})$ away from the diagonal, i.e. $\mathrm{i} \neq \mathrm{j}$

$$
\text { Contrast }=\sum_{i, j=0}^{G-1}(i-j)^{2} p(i, j)
$$

Dissimilarity(DIS):

Similar to GLCM contrast and it is high if the local region has high contract.

$$
\text { Dissimilarity }=\sum_{i, j=0}^{G-1}|i-j| P(i, j)
$$

Entropy(ENT):

This measure the randomness of intensity distribution.

$$
\text { Entropy }=\sum_{i, j=0}^{G-1} p(i, j)(-\operatorname{Inp}(i, j))
$$

Sum of Square Variance(SSV):

$$
\text { Varience }=\sum_{i=0}^{G-1} \sum_{j=0}^{G-1}(i-\mu)^{2} p(i, j)
$$

Sum Average(SA):

$$
\text { aver }=\sum_{i=0}^{2 G-2} i P_{x+y}(i)
$$

Sum Variance(SV):

$$
s v=\sum_{i=0}^{2 G-2}(i-a v e r)^{2} P_{x+y}(i)
$$

Sum Entropy(SENT):

$$
S E N T=-\sum_{i=0}^{2 G-2} p_{x+y}(i) \log \left(p_{x+y}(i)\right)
$$

Difference Variance(DV):

$$
D V=\sum_{i=0}^{G-1}(i-a v e r)^{2} P_{x+y}(i)
$$

Difference Entropy(DENT):

$$
D E N T=-\sum_{i=0}^{G-1} p_{x+y}(i) \log \left(p_{x+y}(i)\right)
$$

\begin{tabular}{|c|c|c|c|c|c|c|c|c|c|}
\hline \multirow{2}{*}{$\begin{array}{c}\text { Various } \\
\text { Styles }\end{array}$} & \multicolumn{7}{|c|}{ Gray Level Co-Occurrence Matrix features } \\
\cline { 2 - 10 } & CON & DIS & ENT & SSV & SA & SV & SENT & DV & DENT \\
\hline$A$ & 0.66 & 0.21 & 1.19 & 48.57 & 12.77 & 168.61 & 1.06 & 0.66 & 0.43 \\
\hline$A$ & 0.84 & 0.24 & 1.41 & 42.53 & 11.47 & 142.93 & 1.25 & 0.84 & 0.46 \\
\hline$A$ & 0.76 & 0.21 & 1.25 & 45.22 & 12.02 & 155.07 & 1.12 & 0.76 & 0.43 \\
\hline $\mathbf{B}$ & 1.06 & 0.30 & 1.39 & 44.65 & 11.91 & 150.43 & 1.23 & 1.06 & 0.54 \\
\hline $\mathbf{B}$ & 0.98 & 0.26 & 1.35 & 42.32 & 11.80 & 146.45 & 1.21 & 0.94 & 0.51 \\
\hline $\mathbf{B}$ & 1.02 & 0.30 & 1.41 & 44.58 & 11.92 & 150.10 & 1.24 & 1.02 & 0.55 \\
\hline $\mathbf{Q}$ & 0.53 & 0.21 & 1.46 & 39.33 & 10.76 & 131.00 & 1.31 & 0.53 & 0.48 \\
\hline $\mathbf{Q}$ & 0.61 & 0.26 & 1.58 & 37.18 & 10.31 & 121.70 & 1.39 & 0.67 & 0.56 \\
\hline $\mathbf{Q}$ & 0.63 & 0.30 & 1.60 & 36.98 & 10.28 & 120.27 & 1.41 & 0.80 & 0.59 \\
\hline $\mathbf{Y}$ & 0.47 & 0.15 & 1.10 & 48.86 & 12.81 & 171.40 & 0.99 & 0.47 & 0.35 \\
\hline $\mathbf{Y}$ & 0.49 & 0.15 & 0.96 & 49.46 & 13.10 & 180.24 & 0.87 & 0.49 & 0.32 \\
\hline $\mathbf{Y}$ & 0.55 & 0.17 & 1.20 & 45.81 & 11.98 & 175.84 & 1.03 & 0.51 & 0.40 \\
\hline
\end{tabular}

Fig. 9. Sample features extracted from GLCM.

\section{SUPPORT VECTOR MACHINE}

SVMs are sets of related supervised learning method for classification and regression. It is a discriminative classifier formally defined by a separating hyper plane. The basic idea is to find a hyper plane which separates the dimensional data perfectly between the various classes 11. SVM simultaneously minimizes the experimental classification error and maximize the geometric margin. So SVM called maximum margin classifiers. Intelligent classifier on the extracted feature vector of all the data samples. In this paper, we have chosen the SVM classifier [12] 13 for training and classification. SVM is a two class classifier that classifies the data samples of two classes by computing maximum margin boundary between them. The separating boundary is expressed in the form of a mathematical optimization problem. In case, the data are nonlinearly separable, we use multi class SVM. In one against one strategy also known as all pairs consists in constructing one SVM for each pairs of classes. In multi class SVMs are trained to pick out the samples of one class from the samples of another class Fig 10. Classification of an unidentified pattern is performed according to the maximum voting for one class 14. In one against all strategy consists of constructing one SVM per class, which is trained to separate the samples of one group from the samples of all remaining groups [15 16]. Classification of an unknown pattern is performed according to the most output among all SVMs. In this paper, non linear SVM, to find a hyper plane that separates the training data by maximal 
margin. 'One against all' in SVM, and hyper planes are implemented, where $\mathrm{n}$ is the number of divisions. Each hyper plane can be used to distinguish one class from the other divisions.

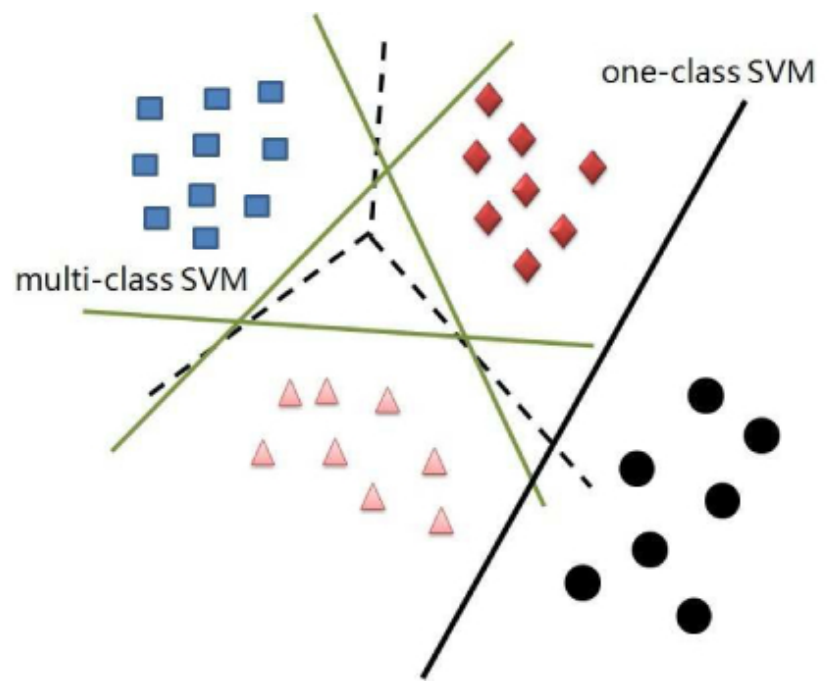

Fig. 10. Multi class SVM.

All the experiments are done on LIBSVM [17] which is multiclass SVM. According to how all the samples can be classified in different classes with suitable margin, different types of kernel in SVM classifier are used. In this experimental work Radial Basis Function (RBF) kernel are used to classify the samples. The effectiveness of SVM depends on kernel parameter gamma(g or $\gamma$ ) and cost parameter c.

\subsection{Kernel Function}

Radial Basis Function (RBF): In RBF kernel, which has a single parameter gamma $(\gamma)$. A Suitable combination of $\mathrm{c}$ and $g$ for optimal result by exponentially rising order,based on parameters $\mathrm{c}$ and $\mathrm{g}$ for each combination.

$$
K\left(x_{i}, x_{j}\right)=\exp \left(-\gamma\left\|x_{i}-x_{j}\right\|^{2}\right), \gamma>0
$$

To determine the value of $\mathrm{g}$ and cost parameter c. These parameters are used in LIBSVM. The parameter is selected in the range of $(0,1)$ for gamma $(\mathrm{g}$ or $\gamma)$ and $(0,1000)$ for cost parameter $\mathrm{c}$ determine the recognition rate.

\section{EXPERIMENAL RESULTS}

The experiments are carried out in Windows 8 operating system with Intel Xeon X3430 Processor $2.40 \mathrm{GHz}$ with 4 GB RAM. The extracted GLCM features are fed to LIBSVM [17 for training. In our experiments 3900 samples of handwritten datasets are collected from 30 different writers. Upper case alphabets written in different styles and sizes are considered. Each writer contributed 5 times of each upper case alphabet out of 26. GLCM feature vector dimensions 9 were extracted from handwritten character. Total 3900 samples handwritten characters were employed for this work. The samples were separated into two divisions, one for training phase and other for testing phase. In training, number of samples were used and measure the performances are shown in Fig 11. Characters are grouped into vertical, horizontal, center and no center as shown in Table 1 based on whether they cross the centroid of the character region segmented.

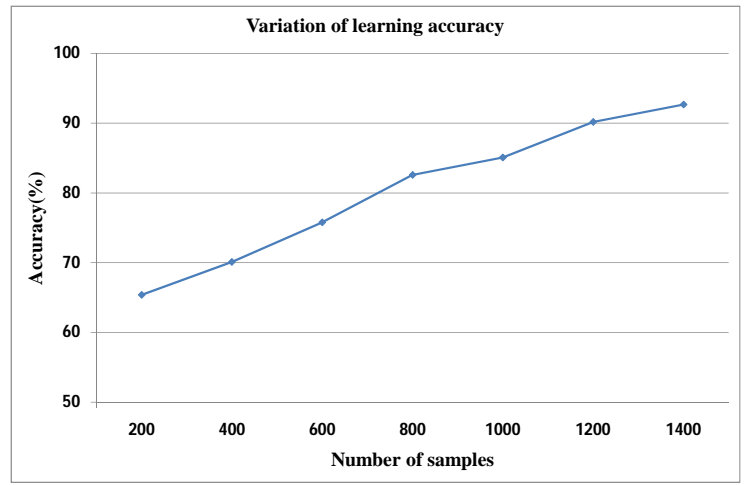

Fig. 11. Number of samples used for training

Table 1. Character Groups

\begin{tabular}{|c|c|c|}
\hline S.No & Groups & Characters \\
\hline 1 & Vertical Position & A,B,H,M,N,P,W \\
2 & Horizontal Position & E,F,G,K,Q,R,S,Z \\
3 & Center Position & I,J,T,X,Y \\
4 & No Center Position & C,D,O,U,V,L \\
\hline
\end{tabular}

In character grouping, 3900 samples are divided into two models (M1, M2), each model contains 1950 samples are based on whether they cross the centroid of the characters are grouped shown in Table 1. M1 model used for training and M2 model used for testing. We obtained recognition accuracy by using RBF kernel various gamma $(\gamma)$ parameter for vertical Fig 12, horizontal Fig 13 , center Fig 14 and no center Fig 15 and combined all groups are shown in Fig 16

\subsection{Evaluation metrics}

Precision $(\mathrm{P})$ and Recall $(\mathrm{R})$ are the commonly used evaluation metrics and these measures are used to evaluate the performance of the proposed system. The measures are defined as follows:

$$
\begin{aligned}
& \text { precision }=\frac{\text { no.of true positives }}{\text { no. of true positives }+ \text { false positives }} \\
& \text { Recall }=\frac{\text { no. of true positives }}{\text { no. of true positives }+ \text { false negatives }}
\end{aligned}
$$

The work used F-score as the combined measure of Precision $(\mathrm{P})$ and recall $(\mathrm{R})$ for calculating accuracy which is defined as follows:

$$
F_{\propto}=\frac{2 P R}{P+R}
$$

where $\propto$ is weighting factor and $\propto=1$ is used. 


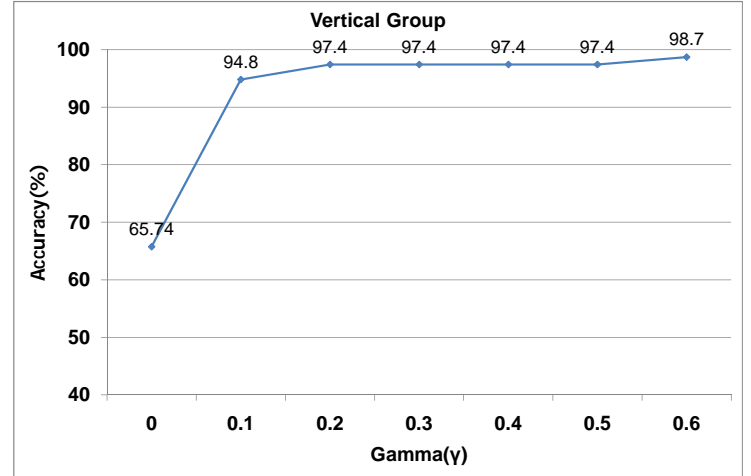

Fig. 12. Recognition accuracy for the vertical group using RBF kernel for various gamma.

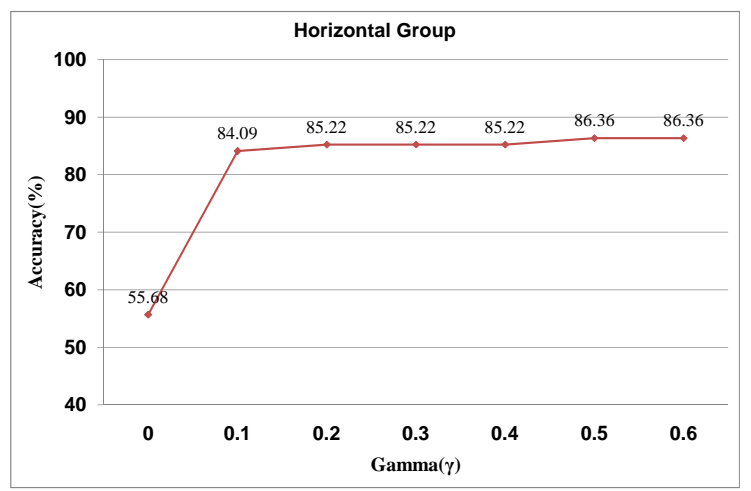

Fig. 13. Recognition accuracy for the horizontal group using RBF kernel for various gamma.

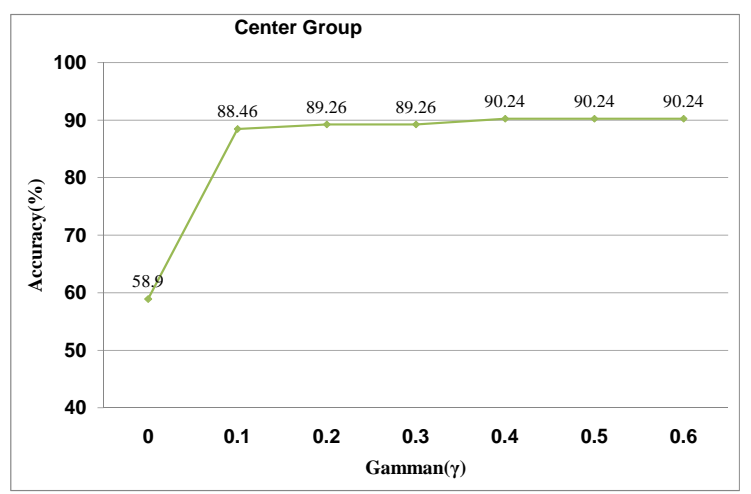

Fig. 14. Recognition accuracy for the center group using RBF kernel for various gamma.

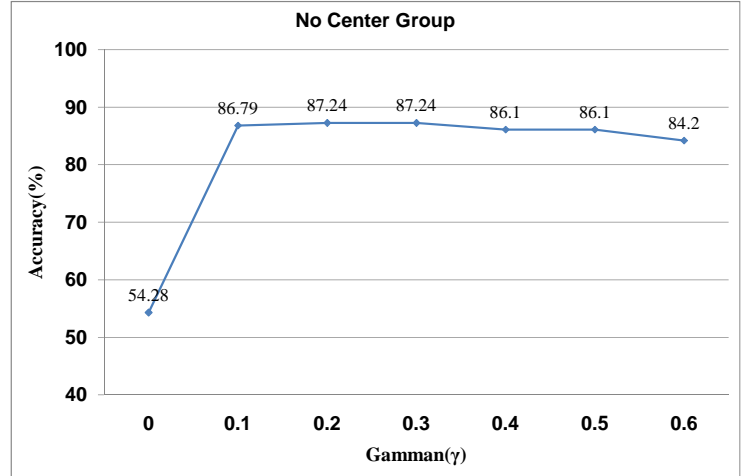

Fig. 15. Recognition accuracy for the no center group using RBF kernel for various gamma.

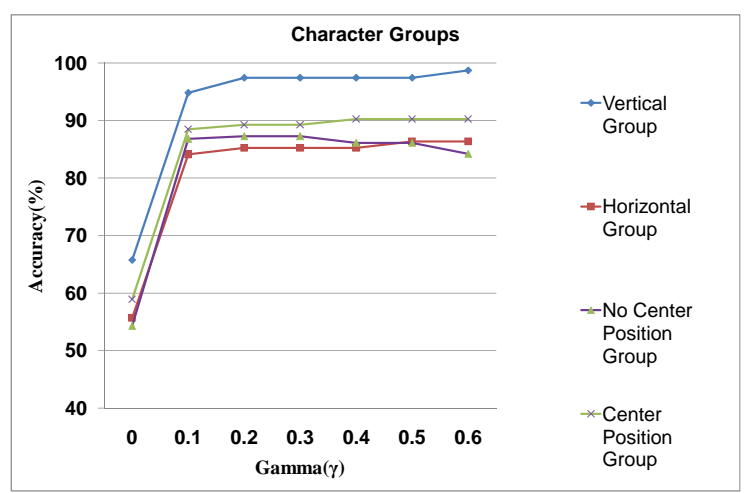

Fig. 16. Recognition accuracy for character groups using RBF kernel for various $\operatorname{gamma}(\gamma)$.

From the total data set, 3000 samples taken for training and 900 samples used for testing. By varying the RBF kernel parameter gamma $(\gamma)$, we obtained minimum recognition accuracy $80.76 \%$ and maximum recognition accuracy $95.43 \%$ as shown in Fig 17

\section{CONCLUSION}

This paper presents an approach for handwritten document recognition using GLCM as features. The approach then evaluates the performance of GLCM features using SVM with RBF kernels. The system given a good classification accuracy $95.2 \%$ by RBF kernel parameter gamma $(\gamma)=0.3$ and cost $(c)=600$. Significant amount of preparation and testing enabled the scheme to have robust recognition accuracy. 


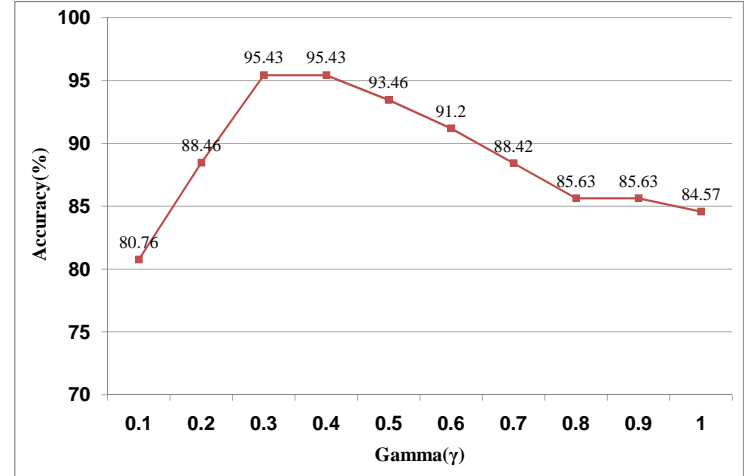

Fig. 17. Recognition accuracy using RBF kernel for various $\operatorname{gamma}(\gamma)$.

\section{REFERENCES}

[1] Pankaj Kumawat, Asha Khatri, Baluram Nagaria. 2013. New approach of hand writing recognition using curvelet transform and invariant statistical features. International Journal of Computer Applications, vol 61-No.18, pp. 2125 .

[2] Gaurav Kumar, Pradeep KumarBhatia. 2013. Neural network based approach for recognition of text images. International Journal of Computer Applications, vol.62No.14, pp. 8-13.

[3] Rajib Lochan Das, Binod Kumar Prasad, Goutam Sanyal. 2012. HMM based offline handwritten writer independent english character recognition using global and local feature extraction. International Journal of Computer Applications, vol. 46-No.10, pp. 45-50.

[4] Mohammed Imrul Jubair, Prianka Banik. 2012. A simplified method for handwritten character recognition from document image. International Journal of Computer Applications, vol.51-No.14, pp.50-54.

[5] Giuseppe Pirlo, Donato Impedovo. 2012. Adaptive membership functions for handwritten character recognition by voronoi-based image zoning. IEEE Transactions on Image Processing, vol.21-No.9, pp.3827-3837.

[6] G.Pirlo, D.Impedovo. 2011. Fuzzy-zoning based classification for handwritten characters. IEEE Transactions on Fuzzy Systems, vol.19-No.4, pp.780-785.

[7] Yusuf Perwej, Ashish Chaturvedi.2011. Machine recognition of handwritten character using neural network. International Journal of Computer Applications, vol.14-No.2, pp.6-9.

[8] Dayashankar Singh, Sanjay Kr.Singh, Dr.Maitreyee Dutta. 2010. Handwritten character recognition using twelve directional feature input and neural network. International Journal of Computer Applications, vol 1-No.3, pp. $82-85$.

[9] Sahar JafarPour, Zahra Sedghi, Mehdi Chehel Amirani. 2012. A robust brain MRI classification with GLCM features, vol.37-No.12, pp 1-5.
[10] Fritz Albregtsen. 2008. Statistical texture measures computed from GLCM, Image processing Laboratory, Dept of Informatics, University of Oslo.

[11] J.P.Lewis, 2004. Tutorial on SVM, CGIT Lab, USC.

[12] D. Elizondo, 2006. The linear separability problem: some testing methods, IEEE Transactions on Neural Networks, vol.17,No. 2.

[13] Nello Cristianini and John Shawe-Taylor, 1999. An introduction to support vector machines and other kernelbased learning methods, Cambridge University Press, New York, NY.

[14] Jonathan Milgram, Mohamed Cheriet, Robert Sabourin. 2006. one against one or one against all which one is better for handwriting recognition with SVM?. Tenth International Workshop on Frontiers in Handwriting Recognition.

[15] Chih Wei Hsu and Chih Jen Lin,2002. A comparison of methods for multi-class support vector machines, IEEE Trans. On Neural Networks, Vol.13,No. 2.

[16] B. Fei, J. Liu,2006. Binary tree of SVM: a new fast multi-class training and classification algorithm, IEEE Transactions on Neural Networks,vol.17,No. 3.

[17] Chih-Chung, Chih-Jen Lin. 2011. LIBSVM:A library for support vector machine, ACM Transactions on Intelligent Systems and Technology, vol.2, pp 1-27. 